\title{
ETERNAL SOLUTIONS TO THE RICCI FLOW
}

\author{
RICHARD S. HAMILTON
}

\section{The result}

We consider solutions to the Ricci flow equation

$$
\frac{\partial}{\partial t} g_{i j}=-2 R_{i j}
$$

on a manifold $X$ of dimension $n$. We say the solution is eternal if it is defined for all time $-\infty<t<\infty$. We are interested in solutions which are complete (which is a way of saying they are also defined for "all" of space) and which have their Riemannian curvature uniformly bounded for all space and time. This is a serious restriction; by the work of W. X. Shi [2] we know then that all the covariant derivatives of the curvature are bounded.

Examples of eternal solutions which are complete with bounded curvature are provided by solitons. These are solutions which move under a one-parameter family of diffeomorphisms. If this comes from exponentiating a vector field $V_{i}$, then we have a soliton when

$$
D_{i} V_{j}+D_{j} V_{i}=2 R_{i j}
$$

since the metric changes by its Lie derivative along the vector field. When the vector field is the gradient of a function we say we have a gradient soliton. If $V_{i}=D_{i} f$, the equation for a gradient soliton is

$$
D_{i} D_{j} f=R_{i j}
$$

so the Ricci tensor is the Hessian of a function. In dimensions 2 and 3 for sure, and probably in all higher dimensions too, there exists a complete gradient soliton with bounded curvature and strictly positive curvature operator which is rotationally symmetric around an origin; it can be found by solving an ODE.

Eternal solutions with bounded curvature are important because they occur as models for slowly forming singularities. Our main result is the following start at a classification.

Received December 4, 1991. Research supported by National Science Foundation grant \#DMS 90-0333. 
1.1. Main theorem. Any complete simply connected eternal solution to the Ricci flow with uniformly bounded curvature and strictly positive curvature operator where the scalar curvature $R$ assumes its maximum is necessarily a gradient soliton.

The proof comes from considering the Harnack inequality for the Ricci flow (see [1]) where we study a quadratic form which vanishes on solitons and is weakly positive on any solution. The condition that $R$ assumes its maximum implies that this quadratic form has a large null space. We can then use the strong maximum principle to see that the only way this can happen is when we are on a soliton.

\section{The Harnack inequality}

In our paper [1] we prove a Harnack inequality for the Ricci flow. There we only assume the solution exists on $0<t<T$, and we derive an estimate with terms $1 / t$ in it. There is an interesting but simple procedure for getting rid of them when our solution is eternal. If we have a solution on $\alpha<t<T$, we can replace $t$ by $t-\alpha$ in the Harnack inequality. Then if $\alpha \rightarrow-\infty$, the expression $1 /(t-\alpha) \rightarrow 0$ and disappears! That proves the following result.

2.1. Theorem. Suppose we have a complete eternal solution to the Ricci flow with uniformly bounded curvature and nonnegative curvature operator. Let

and

$$
M_{a b}=\Delta R_{a b}-\frac{1}{2} D_{a} D_{b} R+2 R_{a c b d} R_{c d}-R_{a c} R_{b c}
$$

and consider the quadratic form

$$
P_{a b c}=D_{a} R_{b c}-D_{b} R_{a c}
$$

$$
Z=M_{a b} W_{a} W_{b}+2 P_{a b c} V_{a b} W_{c}+R_{a b c d} U_{a b} U_{c d}
$$

where $W_{a}$ is a one-form and $U_{a b}$ is a two-form. Then $Z$ is weakly positive, so $Z \geq 0$ for any choice of $W$ and $V$.

(Note we have just dropped the term $\frac{1}{t} R_{a b}$ from the definition of $M_{a b}$ in [1].)

We shall also need the following computations, which are the basis of the proof of the Harnack inequality. They come from [1] by dropping all terms with $1 / t$.

2.2. Computation. At a point where

$$
\begin{gathered}
\left(D_{t}-\Delta\right) W_{a}=0, \quad\left(D_{t}-\Delta\right) U_{a b}=0, \\
D_{a} W_{b}=0 \quad \text { and } \quad D_{a} V_{b c}=\frac{1}{2}\left(R_{a b} W_{c}-R_{a c} W_{b}\right)
\end{gathered}
$$


we have

$$
\begin{aligned}
\left(D_{t}-\Delta\right) Z= & 2 R_{a c b d} M_{c d} W_{a} W_{b}-2 P_{a c d} P_{b d c} W_{a} W_{b} \\
& +8 R_{a d c e} P_{d b e} U_{a b} W_{c}+4 R_{a e c f} R_{b e d f} U_{a b} U_{c d} \\
& +\left[P_{a b c} W_{c}+R_{a b c d} U_{c d}\right]\left[P_{a b e} W_{e} R_{a b e f} U_{e f}\right] .
\end{aligned}
$$

2.3. Computation. At a point where the quadratic form $Z$ can be written as a sum of squares of linear forms

$$
Z=\sum_{M}\left(X_{a}^{M} W_{a}+Y_{a b}^{M} U_{a b}\right)^{2}
$$

the quadratic form

$$
\begin{aligned}
Q= & 2 R_{a c b d} M_{c d} W_{a} W_{b}-2 P_{a c d} P_{b d c} W_{a} W_{b} \\
& +8 R_{a d c e} P_{b d e} U_{a b} W_{c}+4 R_{a e c f} R_{b e d f} U_{a b} U_{c d}
\end{aligned}
$$

is given by

$$
Q=\sum_{M N}\left(Y_{a c}^{M} X_{c}^{N} W_{a}-Y_{a c}^{N} X_{c}^{M} W_{a}-2 Y_{a c}^{M} Y_{b c}^{N} U_{a b}\right)^{2},
$$

which is also a sum of squares of linear forms.

\section{The idea}

We now give the idea of the proof, depending on a lemma which we prove later. The quadratic form $Z$ is defined on the space of $W \oplus U$ in $\wedge^{1} \oplus \wedge^{2}$. If the curvature operator $R_{a b c d} U_{a b} U_{c d}$ is strictly positive, then $Z$ is strictly positive on the subspace $\wedge^{2} \subseteq \wedge^{1} \oplus \wedge^{2}$ where $W=0$. Therefore its null space has dimension at most $\operatorname{dim} \wedge^{1}=n$. If we write $U=V \wedge W$ so that

$$
U_{a b}=\frac{1}{2}\left(V_{a} W_{b}-V_{b} W_{a}\right)
$$

and sum over an orthonormal basis of $W_{a}$ 's

$$
\sum_{W} Z(W \oplus V \wedge W)=\frac{\partial R}{\partial t}+2 D_{a} R \cdot V_{a}+R_{a b} V_{a} V_{b}
$$

and by letting $V=0$ we get

$$
\sum_{W} Z(W \oplus 0)=\frac{\partial R}{\partial t} .
$$

At a point where $R$ assumes its maximum, we will have $\partial R / \partial t=0$, and hence letting $W$ run through a basis $E_{a}$ we have $Z\left(E_{a} \oplus 0\right) \geq 0$ for each 
$a$, so $Z\left(E_{a} \oplus 0\right)=0$ for each $a$. Thus $Z$ has a null space of dimension exactly $n$ at this point.

We then want to argue from the strong maximum principle that any earlier time the null space of $Z$ has dimension $n$ at every point. This is Lemma 4.1. We can then find a smooth tensor $L_{a b c}$ with $L_{a b c}+L_{b a c}=0$ such that for any $W_{a}$ if

$$
U_{a b}=L_{a b c} W_{c}
$$

then $W \oplus U$ is the null space of $Z$. Since the null space of $Z$ satisfies

$$
M_{a c} W_{a}+P_{a b c} U_{a b}=0
$$

and

$$
P_{a b c} W_{c}+R_{a b c d} U_{c d}=0
$$

we find that

$$
M_{c d} L_{a b c} P_{a b d}=0 \text { and } P_{c d e}+R_{a b c d} L_{a b e}=0 .
$$

We can however do even better. Since the null space of $Z$ cannot contract, we see from Computations 2 and 3 that the null space of $Z$ must be contained in the null space of $Q$. Now $W \oplus U$ lies in the null space of $Z$ precisely when

$$
X_{a}^{M} W_{a}+Y_{a b}^{M} U_{a b}=0
$$

for all $M$, and this happens whenever $U_{a b}=L_{a b c} W_{c}$, which shows us

$$
X_{c}^{M}=-Y_{a b}^{M} L_{a b c} .
$$

But then $W \oplus U$ also lies in the null space of $Q$, so

$$
Y_{a c}^{M} X_{c}^{N} W_{a}-Y_{a c}^{N} X_{c}^{M} W_{a}-2 Y_{a c}^{M} Y_{b c}^{N} U_{a b}=0
$$

for all $M$ and $N$. Writing $U$ in terms of $L$ and $W$ and writing $X$ in terms of $L$ and $Y$ and simplifying algebraically give

$$
Y_{e c}^{M} Y_{a b}^{N} L_{a b c}-Y_{e c}^{N} Y_{a b}^{M} L_{a c b}+2 Y_{a c}^{M} Y_{b c}^{N} L_{a b e}=0
$$

for all $M$ and $N$.

Now from the definition of $X$ and $Y$

$$
R_{a b c d}=\sum_{M} Y_{a b}^{M} Y_{c d}^{M}
$$

and since the curvature operator is strictly positive the $Y_{a b}^{M}$ can be chosen as an orthogonal basis for the two-forms. In fact we can take

$$
Y_{a b}^{M}=\lambda^{M} E_{a b}^{M}
$$


where $E_{a b}^{M}$ is an orthonormal basis for the two-forms, and $\left(\lambda^{M}\right)^{2}$ are the eigenvalues of the curvature operator. Then dividing the previous expression by $\lambda^{M} \lambda^{N}$ gives

$$
E_{e c}^{M} E_{a b}^{N} L_{a b c}-E_{e c}^{N} E_{a b}^{M} L_{a b c}+2 E_{a c}^{M} E_{b c}^{N} L_{a b e}=0 .
$$

We now multiply by $E_{p q}^{M} E_{r s}^{N}$ and sum over $M$ and $N$, using

$$
\sum_{M} E_{a b}^{M} E_{c d}^{M}=\frac{1}{2}\left(g_{a c} g_{b d}-g_{a d} g_{b c}\right)
$$

and simplifying to conclude that

$$
\begin{aligned}
& g_{p e} L_{r s q}-g_{q e} L_{r s p}-g_{r e} L_{p q s}-g_{s e} L_{p q r} \\
& \quad+g_{q s} L_{p r e}-g_{q r} L_{p s e}-g_{p s} L_{q r e}+g_{p r} L_{q s e}=0
\end{aligned}
$$

for any choice of $p, q, r, s$, and $e$.

3.1. Lemma. There exists a $V_{a}$ such that

$$
L_{a b c}=\frac{1}{2}\left(V_{a} g_{b c}-V_{b} g_{a c}\right) .
$$

Proof. This follows algebraically from the above relation, and is in fact equivalent to it. To see this, take the trace on $q$ and $s$. This gives

$$
(n-2) L_{p r e}-L_{r e p}-L_{e p r}=X_{p} g_{r e}-X_{r} g_{p e}
$$

when $X_{p}=L_{p q q}$ is the trace. Now we cyclically permute $p, r$, and $e$ and sum to conclude that

$$
(n-4)\left(L_{p r e}+L_{r e p}+L_{e p r}\right)=0
$$

and so

$$
L_{\text {pre }}+L_{r e p}+L_{e p r}=0
$$

at least when $n \neq 4$. It is also true when $n=4$, as we see differently. We only need to check it for $p, r, e$ all distinct, so take $p=1, q=2$, $r=3, s=e=4$ in the original formula and get $L_{123}=0$. Then surely $L_{123}+L_{231}+L_{312}=0$ also, and this is good enough.

Now if we substitute this in the formula above for the trace, we get

$$
(n-1) L_{p r e}=X_{p} g_{r e}-X_{r} g_{p e}
$$

which gives

$$
L_{p r e}=\frac{1}{2}\left(V_{p} g_{r e}-V_{r} g_{p e}\right)
$$

for $V_{p}=\frac{2}{n-1} X_{p}$. This proves the lemma. q.e.d. 
The lemma produces our desired smooth vector field along which the solution is supposed to flow. Substituting back in our formulas for the null space of $Z$ gives

$$
M_{b c}+V_{a} P_{a b c}=0 \quad \text { and } \quad P_{a b c}=R_{a b c d} V_{d} .
$$

Now if we differentiate the second expression and use the first and the relations

$$
M_{b c}=D_{a} P_{a b c}+R_{b d c e} R_{d e} \text { and } D_{a} R_{a b c d}=P_{c d b}
$$

(which hold on any Riemannian manifold) we find that everything simplifies to

$$
R_{a b c d}\left(D_{b} V_{d}-R_{b d}\right)=0 .
$$

Now we let

$$
T_{a b}=D_{a} V_{b}-R_{a b}
$$

and observe that $R_{a b c d} T_{b d}=0$.

3.2. Lemma. The tensor $T_{a b}$ is symmetric.

Proof. Let $T_{a b}=S_{a b}+A_{a b}$ be its decomposition into symmetric and antisymmetric parts. Then

$$
R_{a b c d} T_{b d}=R_{a b c d} S_{b d}+R_{a b c d} A_{b d}
$$

is also such a decomposition. Therefore $R_{a b c d} A_{b d}=0$. Using the Bianchi identity we find that this is equivalent to $R_{a b c d} A_{c d}=0$ and since the curvature operator is strictly positive we must have $A_{c d}=0$, so $T_{a b}$ is symmetric.

In dimension three we could already conclude that $T_{a b}=0$ from $R_{a b c d} T_{b d}=0$. For in a basis where $T_{a b}$ is diagonal we get the equations

$$
\begin{aligned}
& R_{1212} T_{22}+R_{1313} T_{33}=0, \\
& R_{1212} T_{11}+R_{2323} T_{33}=0, \\
& R_{1313} T_{11}+R_{2323} T_{22}=0
\end{aligned}
$$

and the coefficient matrix has determinant

$$
2 R_{1212} R_{1313} R_{2323}>0
$$

so the only solution is

$$
T_{11}=T_{22}=T_{33}=0 .
$$

In higher dimensions this does not suffice, and we have to work harder. A lot harder.

First note that

$$
D_{a} V_{b}=R_{a b}+T_{a b}=D_{b} V_{a}
$$


so that when the manifold is simply connected we can solve globally for a function $f$ with

$$
D_{a} f=V_{a}
$$

We then have the equations

$$
\begin{gathered}
R_{a b c d} T_{b d}=0, \\
D_{a} D_{b} f=R_{a b}+T_{a b}, \\
P_{a b c}=R_{a b c d} D_{d} f, \\
M_{b c}+D_{a} f \cdot P_{a b c}=0
\end{gathered}
$$

and we now work from here. Differentiate (2) and switch derivatives and use (3) to show that

$$
D_{a} T_{b c}=D_{b} T_{a c}
$$

so that $T_{a b}$ is a Codazzi tensor. Now apply the operator $\left(D_{t}-\Delta\right)$ to (2) and let

$$
\left(D_{t}-\Delta\right) f=h
$$

and use the commutation formula

$$
\left(D_{t}-\Delta\right) D_{a} D_{b} f=D_{a} D_{b}\left(D_{t}-\Delta\right) f+2 R_{a c b d} D_{c} D_{d} f
$$

and

$$
\left(D_{t}-\Delta\right) R_{a b}=2 R_{a c b d} R_{c d}
$$

to compute that

$$
\left(D_{t}-\Delta\right) T_{a b}=D_{a} D_{b} h .
$$

If we differentiate (5) again to get

$$
D_{a} D_{b} T_{c d}=D_{a} D_{c} T_{b d}
$$

and switch $D_{a}$ and $D_{b}$ we have

$$
D_{a} D_{c} T_{b d}-D_{b} D_{a} T_{c d}=R_{a b c e} T_{d e}+R_{a b d e} T_{c e}
$$

and then if we cyclically permute $a, b, c$ and sum, and use the Bianchi identity on $R_{a b c e}$, we get

$$
R_{a b d e} T_{c e}+R_{b c d e} T_{a e}+R_{c a d e} T_{b e}=0 .
$$

If we then trace on $a$ and $d$ we get

$$
R_{b e} T_{c e}=R_{c e} T_{b e}
$$

which shows $R_{a b}$ and $T_{a b}$ commute. 
Going back to (7), by the commutator formula

$$
\left(D_{t}-\Delta\right) D_{a} T_{b c}=D_{a}\left(D_{t}-\Delta\right) T_{b c}+2 R_{a d b c} D_{d} T_{e c}+2 R_{a d c e} D_{d} T_{b e}
$$

and this gives

$$
\left(D_{t}-\Delta\right) D_{a} T_{b c}=D_{a} D_{b} D_{c} h+2 R_{a d b e} D_{c} T_{d e}+2 R_{a d c e} D_{b} T_{d e} .
$$

Now switch $a$ and $b$ and subtract. We then have

$$
R_{a b c d} D_{d} h+2 R_{a d c e} D_{b} T_{d e}-2 R_{b d c e} D_{a} T_{d e}=0 .
$$

Going back and differentiating (1) gives

$$
R_{a d c e} D_{b} T_{d e}+T_{d e} D_{b} R_{a d c e}=0
$$

and doing this on the other term also shows that

$$
R_{a b c d} D_{d} h=2 T_{d e}\left(D_{b} R_{a d c e}-D_{a} R_{b d c e}\right)
$$

and by the second Bianchi identity

$$
R_{a b c d} D_{d} h=2 T_{d e} D_{d} R_{a b c e} .
$$

Now from [1] we recall the evolution formulas

$$
\begin{aligned}
\left(D_{t}-\Delta\right) R_{a b c d}= & 2 R_{a e b f} R_{c e d f}-2 R_{a e b f} R_{d e c f} \\
& +2 R_{a e c f} R_{b e d f}-2 R_{a e d f} R_{b e c f}, \\
\left(D_{t}-\Delta\right) P_{a b c}= & 2 R_{a d b e} P_{d e c}+2 R_{a d c e} P_{d b e} \\
& +2 R_{b d c e} P_{a d e}-2 R_{d e} D_{d} R_{a b c e} \\
\left(D_{t}-\Delta\right) M_{a b}= & 2 R_{a c b d} M_{c d}+2 R_{c d}\left[D_{c} P_{d a b}+D_{c} P_{d b a}\right] \\
& +2 P_{a c d} P_{b c d}-4 P_{a c d} P_{b d c}+2 R_{c d} R_{c e} R_{a d b e} .
\end{aligned}
$$

When we apply the evolution operator $\left(D_{t}-\Delta\right)$ to formula $(3)$ there are many cancellations, after which we just recover (9), so we obtain no new information. But if we apply $\left(D_{t}-\Delta\right)$ to (4) and use the given identities, then after many cancellations we are left with one new term! Namely we get

$$
R_{a b c d} T_{b e} T_{d e}=0
$$

which looks just like (1) but is in fact much better since the matrix $T_{a b}$ now is squared.

3.3. Lemma. The tensor $T_{a b}=0$.

Proof. Choose a basis where $T_{a b}$ is diagonal. Then

$$
R_{a b c d} T_{b e} T_{d e}=0
$$


implies that for $a=c=1$

$$
R_{1212} T_{22}^{2}+R_{1313} T_{33}^{2}+\cdots+R_{1 n 1 n} T_{n n}^{2}=0
$$

and since $R_{1212}>0, R_{1313}>0, \cdots, R_{1 n 1 n}>0$ we get $T_{22}=0, T_{33}=$ $0, \cdots, T_{n n}=0$. Similarly all the diagonal entries are zero. q.e.d.

We now have $D_{a} V_{b}=R_{a b}$, so the metric is in fact a Ricci soliton.

\section{The strong maximum principle}

We now prove the lemma we need to make the previous argument work.

4.1. Lemma. If the quadratic form $Z$ has null space of dimension strictly less than $n$ at some point at $t=0$, then it has rank strictly less than $n$ at every point for any time $t>0$.

Proof. This will be a consequence of the usual strong maximum principle, which assures us that if we have a function $F \geq 0$ which solves

$$
\left(D_{t}-\Delta\right) F=0
$$

for $t \geq 0$ and if we have $F>0$ at some point when $t=0$, then we have $F>0$ everywhere as soon as $t>0$.

Suppose now that $Z$ has null space of dimension strictly less than $n$ at some point $X^{0}$ at $t=0$. By picking $X^{0}$ in general position we may assume the dimension of the null space is constant in a neighborhood of $X^{0}$. We can then choose a smooth vector field $Y_{a}$ with support in this neighborhood so that $Y_{a} \neq 0$ at the point $X^{0}$ but $Y_{a}$ is orthogonal to the null space of $Z$, in the sense that if $Z$ vanishes on $W_{a} \oplus U_{b c}$ then $Y_{a} W_{a}=0$. We then define a matrix by

$$
F_{a b}=Y_{a} Y_{b}
$$

at $t=0$, and allow $F_{a b}$ to evolve by the heat equation

$$
\left(D_{t}-\Delta\right) F_{a b}=0 .
$$

Since $F_{a b} \geq 0$ as a matrix at $t=0$, it will remain so for $t \geq 0$ by the maximum principle. Let $F=F_{a a}$ be the trace. Then $F>0$ at the point $X^{0}$ at $t=0$, so $F>0$ everywhere as soon as $t>0$. But this means $F_{a b}$ has rank at least one.

The quadratic form $F_{a b} W_{a} W_{b}$ vanishes on the null space of $Z$, so by multiplying $F_{a b}$ by $\varepsilon>0$ sufficiently small we can arrange things so that $Z \geq F_{a b} W_{a} W_{b}$ at $t=0$. Now we claim that this inequality will be preserved for $t \geq 0$. Whereupon we are done; inasmuch as $R_{a b c d} U_{a b} U_{c d}>$ 0 for $U_{a b} \neq 0$, so if $W_{a} \oplus U_{b c}$ lies in the null space of $Z$ we must have 
$W_{a} \neq 0$, which shows the dimension of the null space of $Z$ cannot exceed the dimension of the null space of $F_{a b}$.

Note that $R_{a c b d} F_{c d} \geq 0$ since $F_{c d} \geq 0$ and $R_{a c b d}$ has positive sectional curvature. Therefore the following result will suffice.

4.2. Theorem. Let $F_{a b}$ be uniformly bounded with $F_{a b} \geq 0$ and satisfy

$$
\left(D_{t}-\Delta\right) F_{a b} \leq 2 R_{a c b d} F_{c d} .
$$

Suppose $Z \geq F_{a b} W_{a} W_{b}$ when $t=0$. Then this remains true for $t \geq 0$.

Proof. We modify the Harnack expression $Z$ by letting

where we let

$$
\widehat{Z}=\widehat{M}_{a b} W_{a} W_{b}+2 P_{a b c} U_{a b} W_{c}+\widehat{R}_{a b c d} U_{a b} U_{c d}
$$

$$
\begin{aligned}
\widehat{M}_{a b} & =M_{a b}-F_{a b}+\varphi g_{a b}, \\
\widehat{R}_{a b c d} & =R_{a b c d}+\frac{1}{2} \psi\left(g_{a c} g_{b d}-g_{a d} g_{b c}\right)
\end{aligned}
$$

where $\varphi$ and $\psi$ are functions we will choose later and then let go to zero. We compute the modifications to the evolution of $\widehat{Z}$ in Computation 2.2 from the introduction of $F_{a b}, \varphi$ and $\psi$. This gives us the following result, using $C$ as a constant to bound $|R m|,|D R m|,\left|D^{2} R m\right|$ and $\left|F_{a b}\right|$, and we assume $\psi \leq 1$.

4.3. Lemma. We have

$$
\begin{aligned}
\left(D_{t}-\Delta\right) \hat{Z}= & 2 \widehat{R}_{a c b d} \widehat{M}_{c d} W_{a} W_{b}-2 P_{a c d} P_{b d c} W_{a} W_{b} \\
& +8 \widehat{R}_{a d c e} P_{d b e} U_{a b} W_{c}+4 \widehat{R}_{a e c f} \widehat{R}_{b e d f} U_{a b} U_{c d} \\
& +\left[P_{a b c} W_{c}+R_{a b c d} U_{c d}\right]\left[P_{a b e} W_{e}+R_{a b e f} U_{e f}\right] \\
& -\left[\left(D_{t}-\Delta\right) F_{a b}-2 R_{a c b d} F_{c d}\right] W_{a} W_{b} \\
& +\left[\left(D_{t}-\Delta\right) \varphi\right]|W|^{2}+\left[D_{t} \psi\right]|U|^{2}-E
\end{aligned}
$$

where the error $E$ is bounded by

$$
E \leq C(\varphi+\psi)|W|^{2}+C \psi|U|^{2}
$$

at a point where

$$
\begin{gathered}
\left(D_{t}-\Delta\right) W_{a}=0, \quad\left(D_{t}-\Delta\right) U_{a b}=0, \\
D_{a} W_{b}=0 \quad \text { and } \quad D_{a} U_{b c}=\frac{1}{2}\left(R_{a b} W_{c}-R_{a c} W_{b}\right) .
\end{gathered}
$$

Proof. Few new terms can occur since we do not have to worry about space derivatives falling on $W_{a}$ or $\psi$. The only trick is to bound the cross-term

$$
C \psi|U||W| \leq C \psi|U|^{2}+C \psi|W|^{2}
$$

which is obvious. 
Now to make the argument work we need to have

$$
\left(D_{t}-\Delta\right) \varphi>C(\varphi+\psi) \text { and } D_{t} \psi>C \psi .
$$

This is easy to achieve. We take $\psi=\delta e^{A t}$ with $\delta$ small and $A>C$. Then we take $\varphi=\delta e^{A t} f(x)$ where $f$ is the function constructed with $f(x) \rightarrow \infty$ as $x \rightarrow \infty$ but all the covariant derivatives of $f$ bounded from Lemma 5.1 in [1], and $A$ is large compared to $C$ before and to $C$ bounding $\Delta f$. We then have $\psi \geq \delta>0$ and $\varphi \rightarrow \infty$ when $X \rightarrow \infty$. Since $M_{a b}, F_{a b}$ and $P_{a b c}$ are bounded while $R_{a b c d}$ is positive, it is clear that $\hat{Z}$ is strictly positive outside of a compact set. If $\hat{Z}$ ever becomes zero, there will be a first time $t^{0}$ this happens, and a point $X^{0}$ and an eigenvector $W_{a}^{0}$ and $U_{a b}^{0}$ where $\hat{Z}$ is zero. Extend $W_{a}$ and $U_{a b}$ to sections with $W_{a}=W_{a}^{0}$ and $U_{a b}=U_{a b}^{0}$ at $\left(X^{0}, t^{0}\right)$ and so that

$$
\begin{gathered}
\left(D_{t}-\Delta\right) W_{a}=0, \quad\left(D_{t}-\Delta\right) U_{b c}=0, \\
D_{a} W_{b}=0 \quad \text { and } D_{a} U_{b c}=\frac{1}{2}\left(R_{a b} W_{c}-R_{a c} W_{b}\right)
\end{gathered}
$$

at $\left(X^{0}, t^{0}\right)$. Then Computation 2.3 shows us that $\left(D_{t}-\Delta\right) \hat{Z}>0$ at $\left(X^{0}, t^{0}\right)$. However $D_{t} \hat{Z} \leq 0$ and $\Delta \widehat{Z} \geq 0$ there, so we have a contradiction. Therefore $\hat{Z}$ can never become zero. We now let $\delta \rightarrow 0$ in the choice of $\varphi$ and $\psi$ (note $A$ remains the same) and we recover the theorem.

\section{References}

[1] R. S. Hamilton, The Harnack estimate for the Ricci flow, J. Differential Geometry 37 (1993) 225-243.

[2] W. X. Shi, Ricci deformation of the metric on complete noncompact Riemannian manifolds, J. Differential Geometry 30 (1989) 303-394.

UNIVERSITY OF CALIFORNIA, LA JOLlA 
